\title{
PROJECT CI-NERGY: TOWARDS AN INTEGRATED ENERGY URBAN PLANNING SYSTEM FROM A DATA MODELLING AND SYSTEM ARCHITECTURE PERSPECTIVE
}

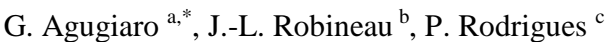 \\ ${ }^{a}$ Austrian Institute of Technology, Smart Cities and Regions Research Field, Vienna, Austria \\ giorgio.agugiaro@ait.ac.at \\ b École Polytechnique Fédérale de Lausanne Valais-Wallis, Sion, Switzerland \\ jean-loup.robineau@epfl.ch \\ ${ }^{c}$ Hochschule für Technik, Dept. Geomatics, Computer Science and Mathematics, Stuttgart, Germany \\ preston.rodrigues@hft-stuttgart.de
}

Commission IV, WG IV/10

KEY WORDS: CityGML, 3D city modelling, Service-oriented infrastructure, Urban Energy Modelling

\begin{abstract}
:
Growing urbanisation, its related environmental impacts, and social inequalities in cities are challenges requiring a holistic urban planning perspective that takes into account the different aspects of sustainable development. One crucial point is to reconcile urban planning with environmental targets, which include decreasing energy demand and $\mathrm{CO}_{2}$ emissions, and increasing the share of renewable energy. Within this context, the project CI-NERGY aims to develop urban energy modelling, simulation and optimisation methods and tools to support decision making in urban planning. However, there are several barriers to the implementation of such tools, such as: fragmentation of involved disciplines, different stakeholders, multiplicity of scales in a city and extreme heterogeneity of data regarding all the processes to be addressed.

Project CI-NERGY aims, among other goals, at overcoming these barriers, and focuses on two case study cities, Geneva in Switzerland and Vienna in Austria.

In particular, project CI-NERGY faces several challenges starting with different cities, heterogeneous data sources and simulation tools, diverse user groups and their individual needs.

This paper describes the experiences gathered during the project. After giving a brief overview of the project, the two case study cities, Geneva and Vienna, are briefly presented, and the focus shifts then on overall system architecture of the project, ranging from urban data modelling topics to the implementation of a Service-Oriented Architecture. Some of the challenges faced, the solutions found, as well some plans for future improvements are described and commented.
\end{abstract}

\section{INTRODUCTION}

There are three main global challenges revolving around cities. The first concerns the growing urbanisation. Today, $54 \%$ of the world population lives in urban areas, and by 2050, an additional 2.5 billion people will be added to the urban population (United Nations, 2014). The challenge here is to provide all the basic services (water, energy, transport, etc.) to everyone in a cost-effective and environmentally friendly way. The second issue are the major environmental impacts that are associated to this urban development. Indeed, cities currently account for $71 \%$ of global energy-related greenhouse gas emissions (GHG) (IEA, 2008), which are contributing to climate change. Moreover, air pollution is responsible for 6.5 million deaths each year (IEA, 2016), mostly due to burning fossil fuels. It is therefore essential that cities reduce their environmental impact. Finally, social inequalities in cities need to be reduced, in part by making services accessible and affordable to all. Facing these challenges requires a holistic urban planning perspective that takes into account the different aspects of sustainable development, namely the economic, environmental and social ones. One of the crucial points is to reconcile urban planning with environmental targets, which include decreasing energy demand and $\mathrm{CO}_{2}$ emissions, and increasing the share of renewable energy.
In this context, the European funded research project "CI-NERGY: Smart cities with sustainable energy systems" aims to develop urban energy modelling, simulation and optimisation methods and tools to support decision making in urban planning [CINERGY]. There are several barriers to implement such tools and applying them. First of all, the disciplines related to the development of those tools are fragmented. Secondly, the various stakeholders involved are usually uncoordinated. Thirdly, the scales of an urban system, going from building to neighbourhood to city level, seem to be irreconcilable. And finally, the lack of data availability and inappropriate data structuring is very often a major issue.

One of the goals of the CI-NERGY project is to overcome these barriers by developing a network of partners from different backgrounds (academia, software developers, urban planners, local authorities, utility companies) and training young scientists in a multi-disciplinary approach. Moreover, two case study cities are used to demonstrate the efficacy of using such models and tools, namely Geneva in Switzerland and Vienna in Austria. A brief overview of the research fields and accomplishments of the project will be given in the following. Buildings are the largest energy-consuming sector in the European Union, accounting for $40 \%$ of total energy use, and offer the largest cost-effective opportunity for savings (Urbact,

Corresponding author 
2013). But identifying where the effort should be put in terms of refurbishment, for instance, requires to have a clear picture of where energy is consumed. Thus, the first focus of the project was to evaluate the heating demand of buildings at a large scale, as the building stock is responsible for the highest share of the energy consumption. To achieve this, different modelling approaches were applied: building simulation using detailed physical models (Monsalvate et al., 2015; Kazas et al., 2017), hybrid approached based on simplified physical models and statistical data (Skarbal et al. 2017), hybrid approaches based on RC models (Goy et al., 2017) and black-box data-driven models using information provided in city-wide building databases (Tardioli et al., 2015).

The development of smart grids is another key aspect of the CI-NERGY project. In this context, an electrical energy demand model for small household appliances was developed. The model takes into account the stochasticity of user behaviour thereby complementing existing models of large load appliances. Furthermore, it can also be combined with a low voltage network model, allowing the evaluation of demand response of residential appliances (Sancho-Tomás et al., 2017). Additionally, demand side management strategies were also evaluated taking into account thermal load management using building capacities (Goy et al., 2017).

Heat supply was also thoroughly investigated. A simulation model of a district heating network was implemented to calculate the energy and exergy performance of different substation configurations (Potente and Ponweiser, 2016). The performance of decentralised solar thermal systems feeding in to district heating networks was also evaluated (Monsalvete et al., 2016). As solar thermal can be combined with seasonal thermal storage, a district energy optimisation model combining both technologies was also developed (Robineau et al., 2016).

In order to fully integrate the different approaches and fields in a holistic approach, co-simulation strategies were investigated (Wang and Robinson, 2016). Finally, a decision support tool, based on optimisation, for early stage planning of sustainable districts was developed (Cajot et al., 2016). This decision support tool combines urban planning and energy supply aspects and provides different options based on the priorities and constraints set by the decision maker. Some of the options are expected to be further evaluated using the set of models developed within the project and mentioned above.

To apply all those models on the case study cities, a substantial effort had to be put into collecting and harmonising heterogeneous data. For this reason, the use of virtual 3D city models for urban energy modelling and planning applications was investigated (Wate and Coors, 2015; Agugiaro, 2016a; Agugiaro, 2016b). Accessibility to the models and the data repositories across the whole consortium was dealt with thanks to web services (Wate et al., 2016).

The goal of this paper is to report and share information about the experiences gathered during the project, especially regarding the data modelling and the general software architecture that were chosen to manage the data and integrate the different tools. The paper will present and describe some of the challenges faced, the solutions found, as well some plans for future improvements.

\subsection{About Geneva}

Geneva is a city in western Switzerland, a centre of trade and finance, home to several international organisations' headquarters, such as the United Nations, and the capital of a Swiss canton that bears the same name. With a population of
201164 inhabitants in 2015, it is the second most populous city in the country, after Zurich, and the centre of a metropolitan area of 946000 inhabitants that extends beyond the borders of the canton. In the CI-NERGY project, the canton of Geneva (490578 inhabitants, $282 \mathrm{~km}^{2}$ ) defines the perimeter of one of the case studies as it is the administrative level at which most datasets are obtained and urban planning decisions made, and therefore from now on "Geneva" will refer to the canton, unless otherwise stated.

The energy strategy of the canton, delegated to the Cantonal Office of Energy (OCEN) [OCEN], is based on two instruments: the General Energy Concept, giving the long-term strategy and goals, and the Energy Directive Plan, translating the orientations into quantified objectives and establishing a list of actions to accomplish them. The long-term goal, aligned with the confederation's one, is to reach a nuclear free "2000-Watt" society by 2050 . This consists in reducing the primary energy consumption to a level corresponding to an average power of 2000 Watts per person over a year, and limiting the $\mathrm{CO}_{2}$ emissions to one ton per person and per year, without reducing quality of life. This level of $\mathrm{CO}_{2}$ emissions corresponds to a consumption of 500 Watts, meaning that the energy supply should be $75 \%$ renewable.

To achieve this goal, several urban and energy planning instruments have been defined by the OCEN, such as the Territorial Energy Concept and the Neighbourhood Directive Plan. The former gives general recommendations to be applied at local scales to improve energy efficiency and increase renewable energy. The latter is a document supporting the development of new neighbourhoods, and which includes a part on energy.

In addition to this, Geneva has 10 major urban development projects, in which energy has an important role. These include both urban regeneration projects of existing areas and greenfield developments of new neighbourhoods. In this context, the CI-NERGY project focused on the development of a decision support tool for urban energy planning, and applied it to one of the greenfield development projects. There is also a will in Geneva to extend the district heating network, and develop new ones, some of which would use the lake as a heat source/sink. Therefore, it is foreseen to apply the district heating network models.

\subsection{About Vienna}

Vienna is the largest city, the capital and, at the same time, one of the nine federal states of Austria with a population, as of 2017 , of over 1.8 million inhabitants (2.6 million within the metropolitan area, 8.7 million in the whole country). Vienna represents the cultural, economic, and political centre of Austria and is the 7th-largest city by population within city limits in the European Union. The city is experiencing a significant population growth: in 2013, its population had increased by almost 200000 persons compared to 2000. According to forecasts, the trend will continue in the coming years, and by 2030 the 2 million inhabitants mark is expected to be achieved. For the entire Viennese agglomeration, which comprises the adjacent districts of Lower Austria and parts of northern Burgenland, the population growth predicted for the same period is of more than 400000 inhabitants. Due to this population growth, in 2011 it was estimated that circa 120000 new dwellings would have to be constructed by 2025 .

As of 2017, there are in Vienna circa 170000 buildings, of which approximately 150000 are of residential or mixed residential type. 
In order to cope with these challenges, a number of planning tools have been adopted since many years, given the long standing urban planning tradition of Vienna. More recently, Vienna has adopted in 2014 the Smart City Wien framework strategy [SC-W], which builds upon and extends the previous Urban Energy Efficiency Programme [SEP-W]. The Smart City Wien framework puts further emphasis on sustainable energy supply by means of conservation and intelligent use of resources. It defines targets for different areas, such as energy efficiency, renewables, infrastructure, mobility, and buildings.

By 2050 Vienna should reach the targets of the "2000-Watt" society, e.g. by decreasing the final energy consumption per capita by $40 \%$ (compared to 2005 ).

More specifically, when it comes to the building sector in a fast growing city like Vienna, a number of measures have already been adopted in the past 20 years with regards to the building rehabilitation (especially in the municipal and social housing sector) or will further be improved in the near future. For existing buildings, a comprehensive rehabilitation is planned in order to reduce the energy consumption for space heating and cooling, domestic hot water production by $1 \%$ per person per year. For all newly built buildings (as well as additions and refurbishments), cost-optimised zero-energy building standards will be enforced from 2018, in order to further boost energy efficiency.

Given the relevance of the building sector in the overall set of energy-related goals defined by the Smart City Wien framework, project CI-NERGY focussed in Vienna particularly on the identification of inefficient (residential) buildings, on the assessment of their energy performance and on the definition of some refurbishment scenarios. In addition, a bottom-up approach was adopted, in order to be able to scale up to block/district scale (whenever required), but keeping the granularity at the single-building level.

\section{CI-NERGY: OVERVIEW, CHALLENGES AND SOLUTION}

The CI-NERGY project aims at developing urban energy modelling, simulation and optimisation methods and tools to support decision making in urban planning. Given the complexity intrinsic to the project, it was therefore of utmost importance to define an integrated system able to cope with the heterogeneity due to:

- Different countries, different cities

- Different geographical scales (building, district, city)

- Different temporal scales (year, months, hours, minutes)

- Different data sources and data formats

- Different tools using such data

in order to a allow for an integrated, shared and replicable environment and avoid technology lock-ins. Therefore, the underlying principles where to focus - as much as (reasonably) possible - on existing free and open solutions, ranging from data models to interfaces, software components, etc.

The first part of this section focuses primarily on the data modelling aspects while the next one deals with the overall system architecture. The two test areas in Geneva and in Vienna are also briefly described.

\subsection{Urban data model}

In order to tackle the heterogeneity mentioned before, what is needed is an integrated city model able to represent coherently all city entities, spatially and (possibly) in $3 \mathrm{D}$, together with all relevant characteristics, relations and dependencies. What is more, the city model should be based on open standards, offer clear data structures, ontologies and semantics in order to facilitate data exchange between different domains and applications. In short, such a standard-based semantic 3D city model would represent a common information hub for integrated urban planning and multi-domain applications, in other words a sort of "Urban Information Model" (UIM), analogous to the otherwise well-known Building Information Model (BIM).

When it comes to urban data models, the Open Geospatial Consortium's CityGML standard is so far the only existing open standard which is mature enough and has enough flexibility to cover the needs of the CI-NERGY project. What is more, it guarantees functional overlaps both with the BIM domain, given its semantic and spatial granularity which for the buildings reaches down till the room level, and (at least in Europe) with the upcoming INSPIRE Data Specification on Buildings when it comes to the regional/national/international level [INSP-BDG].

What is more, CityGML was conceived specifically to model all relevant urban thematic area and entities (buildings, terrain, traffic, vegetation, etc.), and it includes topology, appearance and semantics. A considerable asset of CityGML is its flexibility to model an object with different Levels of Details, enabling the virtual city model to adapt to local building parameter availability and application requirements. Since CityGML is an application independent information model, it does not include domain specific elements by default. However, with the CityGML concept of Application Domain Extensions (ADE), it is possible to incorporate domain-specific entities which are not pre-defined in CityGML standard, like the recent UtilityNetwork ADE [UTN-ADE] which allows for modelling gas, wastewater, district heating/cooling networks or electricity grids with 3D topographic city objects, or the Energy ADE [ENE-ADE], which extends CityGML by a number of energyrelated features and attributes in order to perform energy simulations at city scale but with the granularity which reached down to the single building.

Finally, there already exist a number of free and open tools (database, 3D visualisation tools, import/export tools) which reduce the implementation effort avoiding to start from scratch. For the above mentioned reasons, CityGML was adopted in the CI-NERGY project as the shared urban data model for both cities. Both for Geneva and Vienna, a number of input datasets were gathered, before being further harmonised and integrated in order to obtain for each city a semantic 3D city model based on CityGML and acting as the centralised information hub upon which the other applications were built.

For both cities, for example, the free and open-source 3D City Database tools were adopted and further extended.

\subsubsection{Geneva}

Geneva is very advanced in terms of publicly available datasets regarding the canton, including energy and building related data. The SITG online-platform (Système d'Information du Territoire à Genève) [SITG] provides over 500 geo-referenced datasets in different raster and vector formats, many of which are publicly available.

All building-related data are linked to a unique building identifier called EGID, which allows identifying a building uniquely in the whole of Switzerland. This greatly simplifies the integration of different datasets. Information on buildings include building usage, construction/renovation period, number of floors and annual heating demand. The latter is available for all non-residential buildings and buildings with 5 dwellings or more. Unfortunately, it is uncommon for smaller residential buildings to have this information. 
Some datasets are linked to the address, notably sociodemographic information (e.g. number of inhabitants) and energy consumption data (electricity, gas, district heating) obtained from the local utility service company Services Industriels de Genève (SIG). Each address also has a unique identifier called IDPADR. An important asset of the Geneva case study, is that all address IDs (IDPADR) can be assigned to the respective building IDs (EGID) to which they belong. Data available at address level can therefore be easily aggregated (several addresses can be associated to the same building) to obtain it at building level.

Energy-related data are generally aggregated as annual sum, while more detailed profiles are not easily available. Nevertheless, detailed electric load profiles at 15-minute timesteps were available for almost 7000 clients (out of 274000) which are on the non-regulated market. These mostly concern "big" consumers such as industries, office and administrative buildings, or large residential buildings. Moreover, hourly substation data including thermal load and supply/return temperatures were provided by SIG for around 50 sub-stations. Also, the layout of the networks (electricity, gas, district heating, water) was provided for the whole canton.

Finally, the LoD2-geometries of all buildings in the canton were available and were semantically enriched with all the relevant data mentioned above.

An excerpt of the integrated semantic 3D city model of Geneva is shown in Figure 1 and can be retrieved at: http://scistifmsrv2.epfl.ch:8080/projects/cinergy/geneva/cesium/ web3d_glb/3dwebclient/index.html

\subsubsection{Vienna}

In Vienna, attention was paid in order to identify a test area which would represent a good compromise in terms of available data, heterogeneity in terms of buildings, and size (both geographical extension and number of buildings). As a result, a test area corresponding to the $12^{\text {th }}$ district, Meidling, was chosen. The district of Meidling spans an area of approximately $8.2 \mathrm{~km}^{2}$, it counts circa 90000 inhabitants (i.e. circa 11000 inhabitants $/ \mathrm{km}^{2}$ ) and is a densely populated urban area with numerous residential buildings of different sizes and typologies, but also with large recreational areas and parks. It can be approximately divided into two main parts: the north-eastern one is characterised by a heavily developed urban residential texture, while the south-western one is a more mixed (industrial and light residential) area, which then gradually continues southwards. In Meidling there are circa 7400 buildings, of which approximately 5600 are residential or mixed-residential ones.

Starting from 2015, a large number of datasets (both spatial and non-spatial) were collected for the whole city of Vienna, mainly from the already available Open Government Data Wien catalogue [OGD-W], or were kindly provided by the Municipality of Vienna for the purposes of the CI-NERGY project.

Most of the collected datasets contain information about the building stock, although at different levels of detail and completeness. For example, spatial information of the buildings is available in different datasets up to LoD2, and thematic information (addresses, number of storeys, usage, etc.) is spread over a number of other datasets. Regardless of the scale, the same applies when it comes to energy-related datasets, e.g. in the case of installed photovoltaic and thermal systems, groundwater or wind potential cadastre, etc. A detailed list of the collected data can be found in (Agugiaro, 2016a).

Unlike in the case of Geneva, a conceptually similar unique ID like the EGID was missing, so the data integration steps resulted in a less straightforward progress. A detailed description of all required steps in order to create a 3D virtual city model similar to the one used in Geneva would be far beyond the scope of this paper. Nevertheless, more details can be found in Agugiaro (2016a) about the most relevant data integration issues, and in Agugiaro (2016b) regarding more specific issues regarding energy-related datasets.

An excerpt of the integrated semantic 3D city model of Geneva is shown in Figure 2 and can be retrieved at: http://sbc1.ait.ac.at/projects/meidling/cesium/webmap/index.ht $\mathrm{ml}$

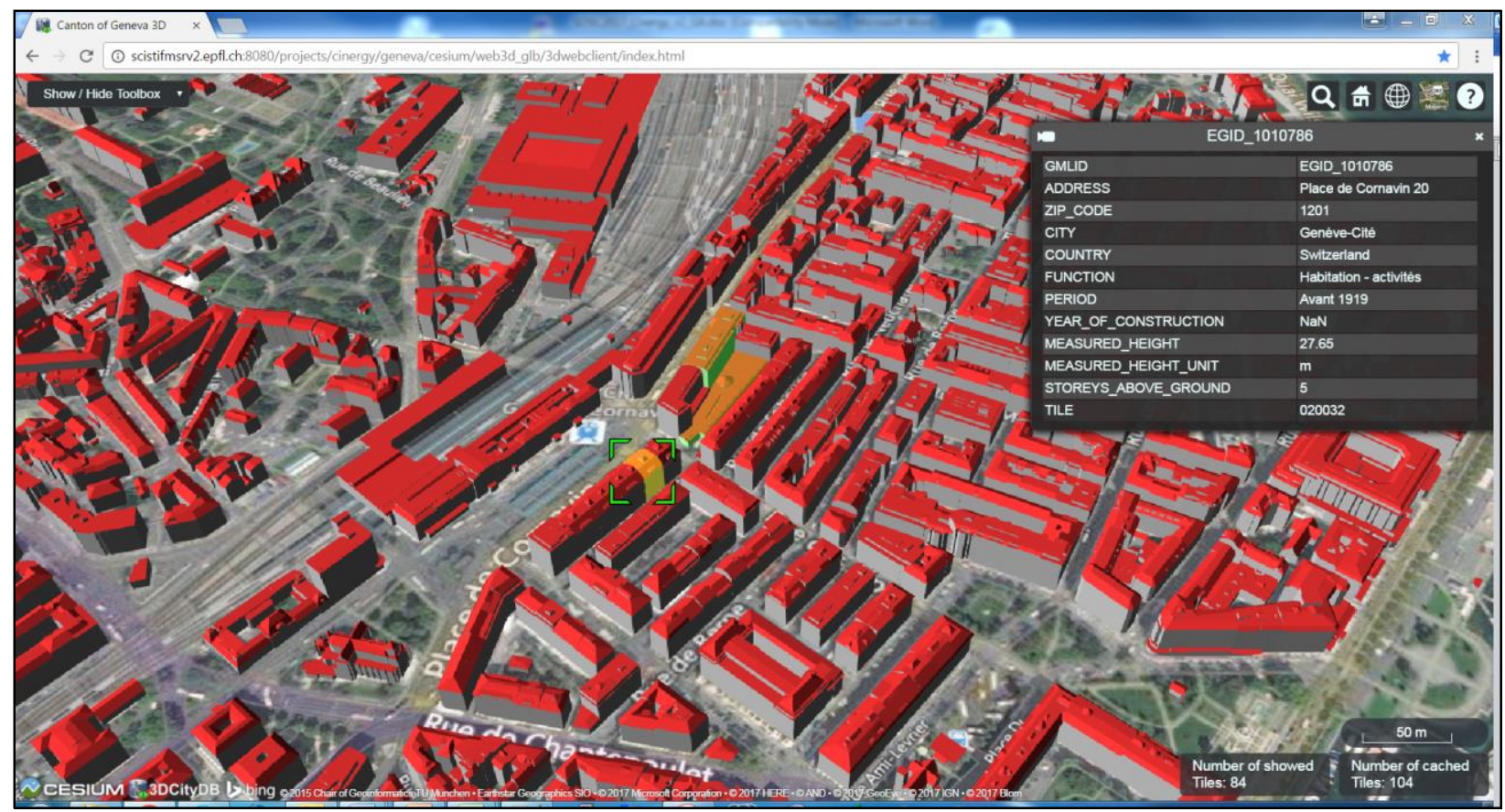

Figure 1 Web-based 3D visualisation of the 3D city model of the Canton of Geneva. For each building, here represented in LoD2, a number of attributes are available. Some of them can be retrieved directly by clicking on the selected object 


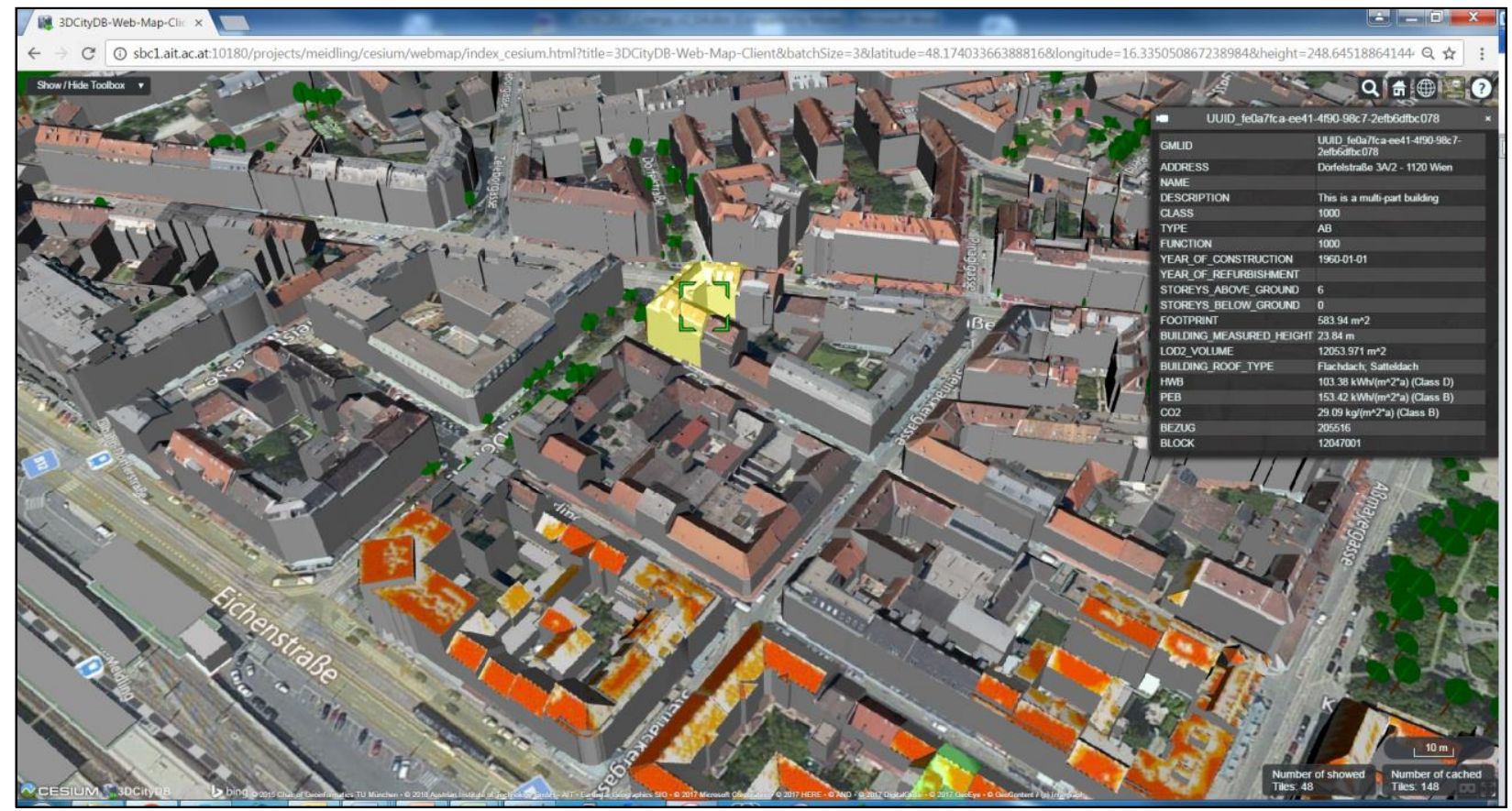

Figure 2 Web-based 3D visualisation (in LoD2) of the semantic 3D city model of the district of Meidling in Vienna. Different textures are visible: roof textures are either from the orthophoto or from the solar cadastre of Vienna. As for Geneva, a number of attributes are available for each city object (e.g. buildings and trees)

\subsection{Service-Oriented Architecture}

Service-Oriented Architecture (SOA) is the computing paradigm that acts as an enabler to expose software functionalities/applications as web services. As shown in Figure 3 , a SOA is made up of three core entities, namely the Service Provider, the Service Consumer and the Service Registry.

The Service Provider is an entity that is willing to provide a software functionality. The Service Consumer is an entity that needs to use a software functionality. The Service Registry is an entity that enables Service Provider to publish its software functionality and allows the Service Consumer to search for a software functionality that it requires to fulfil a task.

Each of these entities relies on key standard technologies (i.e. SOAP, WSDL, UDDI). Simple Object Access Protocol (SOAP) (Gudgin et al., 2007) defines a messaging standard based on Extensible Markup Language (XML) and, with the help of Hypertext Transfer Protocol (HTTP), provides a communication protocol for accessing web services. Web Service Description Language (WSDL) (Christensen et al., 2001) is used to describe the web service access interface. Universal Discovery, Description and Integration (UDDI) (Clement et al., 2004) is a

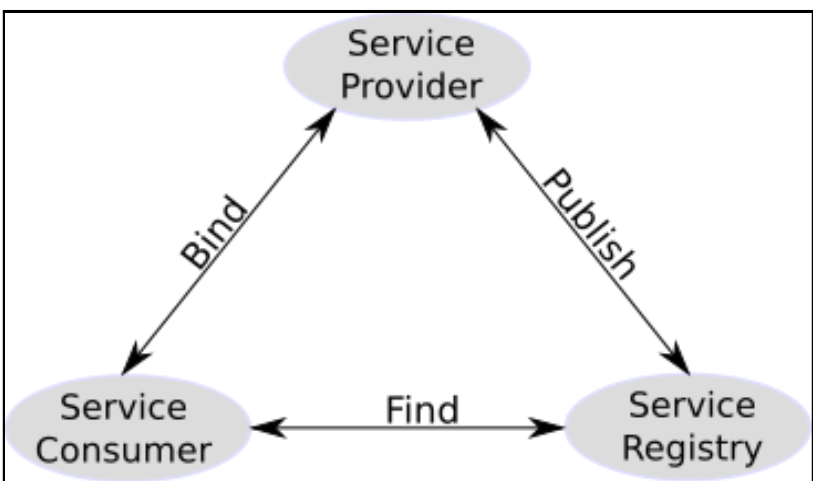

Figure 3 Representation of the three core entities and their relations in a Service-Oriented Architecture registry that allows advertisement and discovery of web services thereby providing the opportunity to dynamically bind a web service at runtime.

Traditionally, Geographic Information Systems (GIS) were stand-alone systems used to store, query, visualise, and analyse geographically referenced information. However, with the recent advancements in Information and Communication Technology it is now possible to store, process and exchange large amount of data seamlessly in a distributed environment. This has enabled the GIS community to rely on well-known industry concept of SOA. Furthermore, the use of web services provide the necessary flexibility to access data and computing resources by different GIS entities. Moreover, it enables interoperability among distributed GIS resources. Realising the numerous advantages of web services, the Open Geospatial Consortium is leading and coordinating the efforts of international organisations and enterprises to develop interoperable GIS services to access distributed geospatial resources. Towards this effort, a series of standard based interface specifications are already available, namely Web Feature Service (WFS), Web Map Service (WMS), Web Coverage Service (WCS), Catalogue Service for the Web (CSW), and Web Processing Service (WPS). Furthermore, in order to enable seamless integration of OGC services, WPS is designed to act as a glue. It is used to orchestrate complex geocomputation processes and their execution mechanism along with their associated inputs and outputs.

One of the most important outcomes from the combination of geospatial information and Internet of Things (IoT) is the creation of an unprecedented amount of data. Storage, ownership and processing of this data has thus become a critical issue. Since most cities own their city data (e.g. building registers, census, cadastre, CityGML data, etc.), storage of this data outside the city/country might not be possible or even allowed due to privacy or data protection reasons. However, thanks to the implementation of web services, one can access different data sources from the city without the need to store the data on external servers, thus overcoming a number of potential 
data-related issues. Furthermore, with decreasing hardware and software prices it is relatively easy to purchase computational resources to process and simulate large amount of geospatial data on-the-fly. The seamless integration of all these entities (data, storage, processes, etc.) by means of a SOA plays therefore a major role in complex environments where a traditional stand-alone approach cannot suffice anymore.

As a matter of fact, this corresponds very well to the case of project CI-NERGY, as all the aforementioned constraints apply. For this reason it was decided to adopt a Service-Oriented Architecture and to proceed with the design of individual geospatial web services for each functionality (data access, processing and simulation). Furthermore, the Business Process Execution Language (BPEL) (Alves et al., 2007) was adopted to design geospatial workflows by integrating individual web services into end-to-end processes. Moreover, since BPEL supports flow control, Sync and Async Transactions as well as data manipulation using XPath, XSLT, and XQuery, it was used to automate and execute user-defined geospatial workflows at runtime.

\subsubsection{CI-NERGY distributed architecture}

As mentioned before, the CI-NERGY project relies on web services in order to accomplish different tasks. Figure 4 illustrates the distributed services in CI-NERGY along with their host locations. Linux servers running Tomcat and Java 8 are hosted at three locations in Europe: at EPFL in Lausanne (Switzerland), at AIT in Vienna (Austria) and at HFT in Stuttgart (Germany). In order to host the web services on these servers, the Apache Axis2 Webservice Framework is adopted. Furthermore, the user can design custom geospatial workflows with the help of the Apache ODE BPEL Framework. Finally, as mentioned in section 2.1, the CityGML-based 3D city models of both cities are stored in two separate PostgreSQL/PostGIS servers which implement each an instance of the 3DCityDB.

Currently, several web services have been implemented, such as WFS, Preprocessing Service, Building Usage Library Service, Construction Library Service, Weather Library Service and Simulation Service. They are briefly described in the following.
- Two WFS are used to retrieve CityGML files of either cities using the GMLID of a building (or by defining a bounding box) and using the CityGML attributes like Year of Construction, Building Function, Roof Type, etc.

- The Construction Library Service provides properties and physical parameters about wall construction like Thickness, Density, Heat Capacity and Conductivity. This information can be retrieved for each layer used for a particular wall type (roof, ground, internal wall and external wall). Additionally, it also provides parameters for glazed surfaces like $u$ values, $g$ values, etc.

- The Building Usage Library Service provides information about the building's internal gains (due to people, electrical appliances and lighting), the convective, radiant and latent fraction with hourly schedules for different profiles (weekday, weekend, holiday, summer and winter designday). Additionally, it provides hourly schedules for space heating/cooling, domestic hot water production, electrical appliances and ventilation. Currently the following building usage types are supported: administrative, apartment building, meeting venues, hospital, industry, restaurant, sales, schools, single-family home, sport installation, swimming pool and warehouse.

- The Weather Service provides all necessary weather data needed to perform the simulations in hourly resolution;

- The Simulation Service takes care of running the actual simulation once all input data and parameters have been retrieved. Furthermore, depending on the chosen simulation template it is also possible to update simulation parameter.

Thanks to the combination of each of these services the user can orchestrate user-defined geospatial workflows.

The current implementation of the platform supports and has been successfully tested with three simulation templates, namely: Irradiation on tilted surfaces, PV potential of roof surfaces and Monthly energy balance.

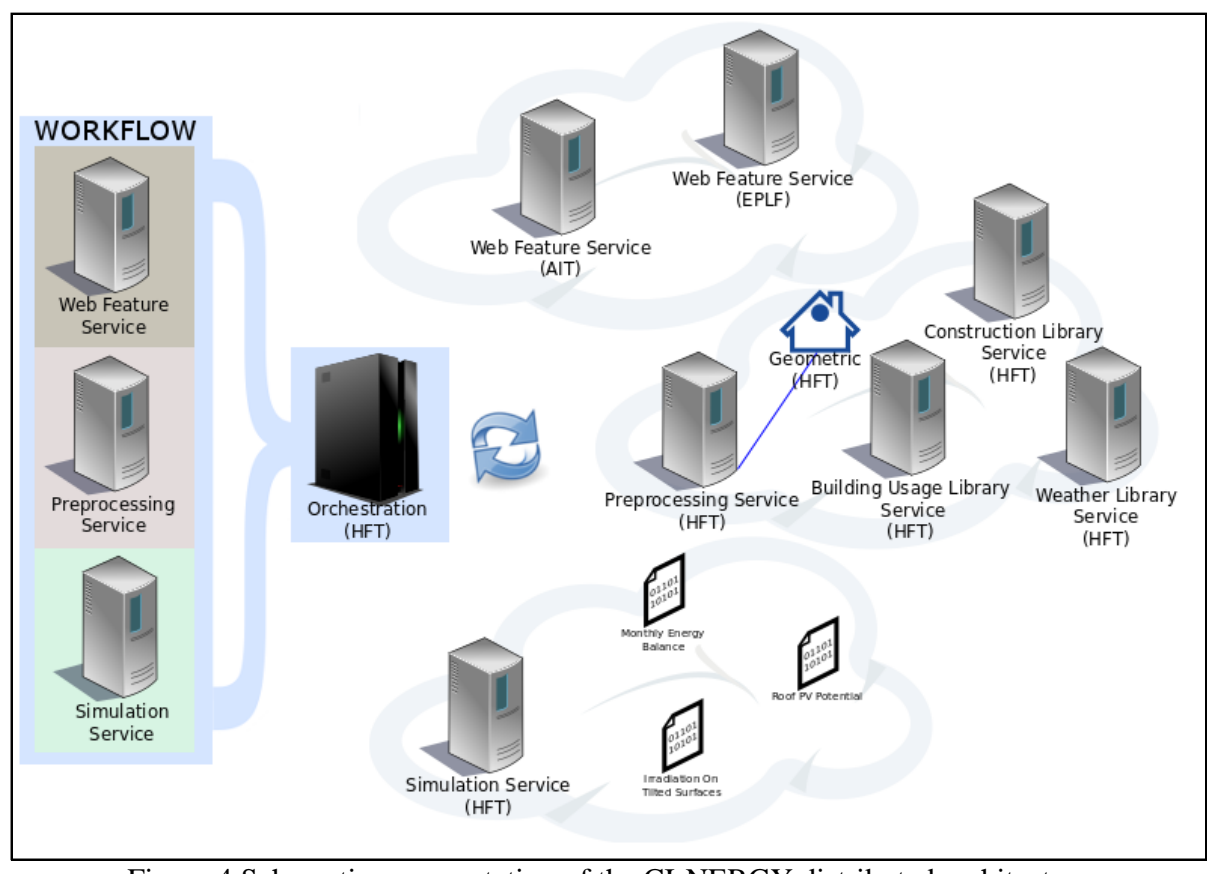

Figure 4 Schematic representation of the CI-NERGY distributed architecture 


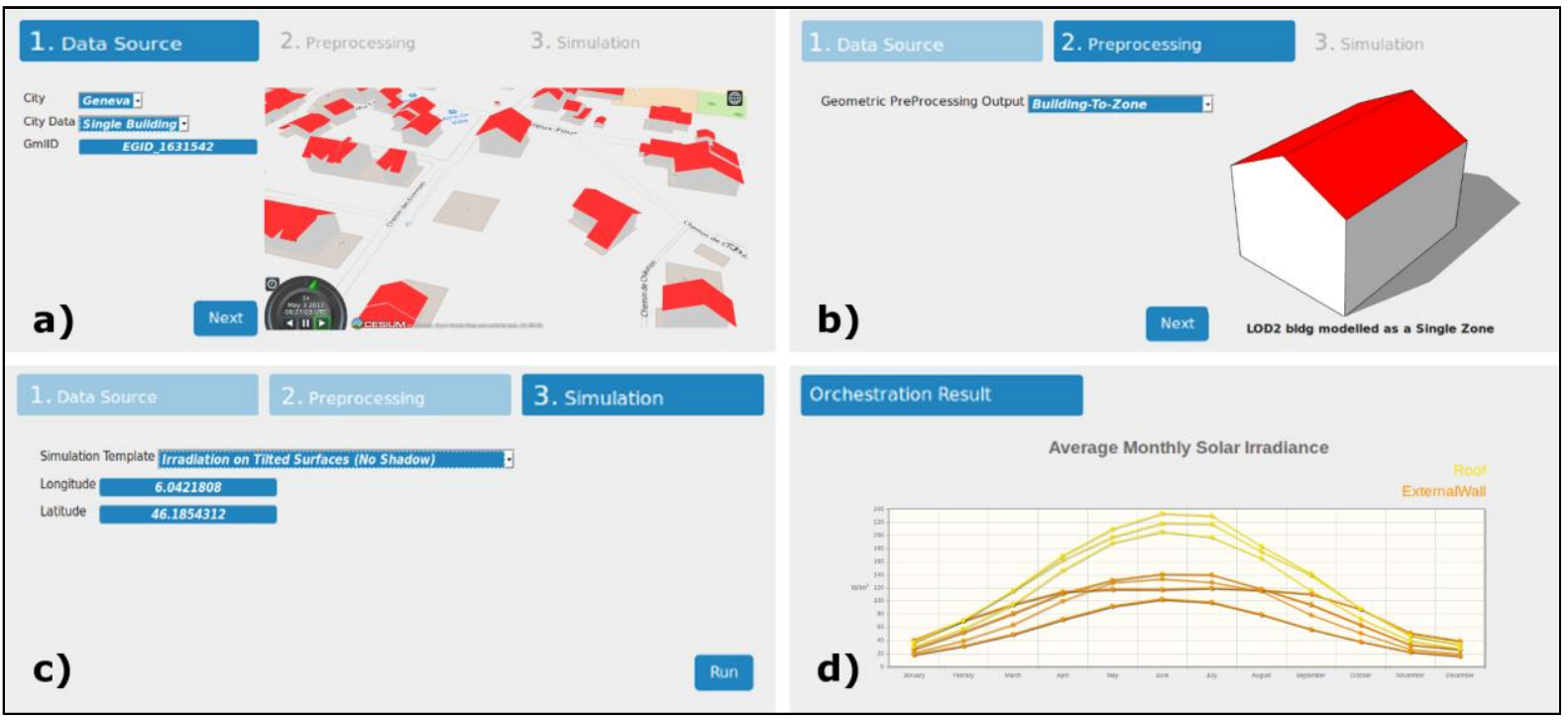

Figure 5 Exemplary workflow in CI-NERGY Platform to compute Irradiation on tilted surface. The user selects the desired building(s) (a), choses the pre-processing task to be carried out (b) and finally the type of simulation (c). Results are finally presented (d).

\subsubsection{Proof-of-concept prototype}

One of the key design objectives of the CI-NERGY project is to describe and automate geospatial workflows using web service technologies and to provide an easy-to-handle user interface. Keeping these objectives in mind, a prototype of CI-NERGY platform was designed and implemented. An example can be retrieved at http://dmz02.rz.hft-stuttgart.de:8080/CINERGY1.

The platform enables a user to interactively define custom geospatial workflows within the three following tasks:

a) Choose a $3 \mathrm{D}$ city data source;

b) Choose a pre-processor for the 3D data source;

c) Choose a simulation template.

Every task can have a number of sub-tasks. In order to complete these tasks, the user needs first of all to access and explore the 3D city models in order to select the building(s) to consider for the successive simulation process. This is accomplished by means of the Cesium WebGL Globe library, which is embedded in the platform GUI to provide an intuitive 3D visualisation interface. Figure 5a shows the first workflow task (Data Source), which consists of different steps. Here the user has to select first the city and then the 3D building(s). This is accomplished by setting whether a simulation on a Single Building or a Region is desired, and by choosing the appropriate option accordingly. Finally, the user chooses a 3D Building by simply clicking on the desired building to consider for simulation. In this example, the user chooses to do a simulation on a Single building: the GMLID of the selected building is displayed to confirm the selection. Once this task is complete the user proceeds towards the next task (Pre-processing).

The Pre-processing task calculates geometric parameters for the selected building(s). It takes a CityGML files as an input and generates several output formats, namely CSV, Building-toZone and Building_storey-to-Zone. Using the GMLID selected from the previous task, a CityGML file is retrieved from the corresponding 3DCityDB by WFS. As shown in Figure 5b, the user chooses here Building-to-Zone. The output of the preprocessing task is passed as an input to the Simulation task. In this example (Figure 5c), the user chooses Irradiation on tilted surface. This simulation template does not require any further user inputs as all data required for this template are already provided from the previous task. Finally, the results of the simulation are shown as a plot (Figure 5d). Alternatively, the result values can be visualized on the selected 3D building as well.

As described, the CI-NERGY platform is highly customisable: the simulation results are calculated based on the users' requirements, i.e. depending on the selected time step. Furthermore, the platform does not define a fixed graphic representation, in that the user is free to visualise the results in the graphic representation and time resolution they prefer.

As the platform is evolving, several individual workflows are being regularly updated. In the future, the plan is to add more flexibility to the platform, which would enable the user to add and remove individual workflow steps on-the-fly.

\section{CONCLUSIONS AND OUTLOOK}

Sustainable development of cities is necessary to face challenges such as climate change, air pollution and poverty. With energy being at the heart of many of these issues, urban planning needs to go hand-in-hand with energy planning. Urban energy modelling, simulation and optimisation methods and tools appear to be a key component in finding promising solutions. In this context, project CI-NERGY has proposed an integrated approach which deals with the aforementioned topics and tackles the complexity of such holistic approaches. In particular, project CI-NERGY is facing heterogeneity starting from different cities in different countries, different data sources, different simulation tools, and different users and needs.

After giving a brief overview of the CI-NERGY project, this paper has presented the two case study cities, Geneva and Vienna and focussed on the overall system architecture of the project, ranging from urban data modelling to the implemented Service-Oriented Architecture.

From the data modelling point of view, the adoption of the CityGML standard for urban data modelling has granted an open, powerful, flexible and extendible solution which allows to provide harmonised and homogeneous data to the following simulation tools, regardless of the original data formats and origin. What is more, the extendibility of CityGML by means of Application Domain Extensions (e.g. Energy and UtilityNetwork ADE) has greatly improved the modelling 
capabilities of CityGML for energy-related tools. At the same time, there has been a mutual benefit also for the ADE development communities, as the CI-NERGY project has actively contributed to their further refinement, development and implementation.

In this paper, an integrated approach for urban energy simulation was introduced. The approach uses the well-known industry concept of Service-Oriented Architecture. A proof-ofconcept prototype was developed to demonstrate the feasibility of the approach by orchestrating services in a distributed environment. The current implementation only supports CityGML LoD2 models, as both case studies' city models are available only up to LoD2, although for urban planners or energy scientist LoD3 gives a more realistic representation of the buildings. Nevertheless, in future it is already planned to extend the simulation platform's support to LoD3 and LoD4 models. Furthermore, integration of additional simulation templates is also planned. Currently, work is being carried out to add templates for dynamic simulations that supports for example results in hourly resolution. Moreover, the mid-tolong-term goal is to extend the approach to address Optimization As a Service (OAAS).

In conclusion, one of the goals of project CI-NERGY was to overcome the barriers that normally exist between disciplines which are related to urban planning and urban energy modelling. Holistic urban planning by means of integration of models and data using web services can contribute to help coping with the diversity of actors, interests, objectives, political targets, thus bringing closer traditionally fragmented disciplines and tools. The final goal is indeed to support planners to coordinate different points of views and political targets.

As a last point, it is worth noting that some of the results of project CI-NERGY are being already exploited by a new project, namely IntegrCiTy [IntegrCiTy], which specifically takes advantage of some of the experiences and results obtained so far in Geneva although it focuses mostly on multi-energy networks optimisations, but is based for example on the same data model.

\section{ACKNOWLEDGEMENTS}

The authors gratefully acknowledges the European Commission for providing financial support during the conduct of research under the FP7-PEOPLE-2013 Marie Curie Initial Training Network CI-NERGY project with Grant Agreement Number 606851. As indicated in section 2, some of the datasets used in this work were kindly provided by the City of Vienna, the Canton of Geneva and Services Industriels de Genève specifically for the CI-NERGY project.

\section{REFERENCES}

Agugiaro, G., 2016a, First steps towards an integrated CityGML-based 3D model of Vienna. ISPRS Ann. Photogramm. Remote Sens. Spatial Inf. Sci., III-4, pp. 139-146.

Agugiaro, G., 2016b, Enabling "energy-awareness" in the semantic 3D city model of Vienna. ISPRS Ann. Photogramm. Remote Sens. Spatial Inf. Sci., IV-4-W1, pp. 81-88.

Alves, A., Arkin, A., Askary, S., Barreto, C., Bloch, B., Curbera, F., 2007, Web Services Business Process Execution Language v. 2.0

Cajot, S., Schüler, N., Peter, M., Page, J., Koch, A., Maréchal, F., 2016, Establishing links for the planning of sustainable districts. Sustainable Built Environment (SBE) Regional Conference, Zürich.

Clement, L., Hately, A., von Riegen, C., Rogers, T., 2004, Universal Description Discovery and Integration (UDDI) v. 3.0.2
Christensen, E., Curbera, F., Meredith, G., Weerawarana, S., 2001, Web Services Description Language (WSDL) 1.1.

Goy, S., Ashouri, A., Maréchal, F., Finn, D., 2017, Estimating the Potential for Thermal Load Management in Buildings at a Large Scale: Overcoming Challenges Towards a Replicable Methodology. Energy Procedia, vol. 111, pp. 740-749.

Gudgin, M., Hadley, M., Mendelsohn, N., Moreau, J.-J., Karmarkar, A., Lafon, Y., 2007. Simple Object Access Protocol (SOAP) v. 1.2 part 0 : Primer ( $2^{\text {nd }}$ edition).

IEA, 2008, World Energy Outlook 2008. International Energy Agency. IEA, 2016, World Energy Outlook Special Report - Energy and Air Pollution. International Energy Agency.

Kazas, G., Fabrizio, E., Perino, M., 2017, Energy demand profile generation with detailed time resolution at an urban district scale: A reference building approach and case study. Appl. Energy, vol. 193 , pp. 243-262.

Monsalvete, P., Eicker, U., Robinson, D., 2016, Energy performance of decentralised solar thermal feed-in to district heating networks. $15^{\text {th }}$ Int. Symposium on District Heating and Cooling, Seoul, South Korea.

Monsalvete, P., Robinson, D., Eicker, U., 2015, Dynamic Simulation Methodologies for Urban Energy Demand. Energy Procedia, vol. 78, pp. 3360-3365.

Potente, M., Ponweiser, K., 2016, Simulation of a district heating network for a case study in the city of Vienna considering energy and exergy losses. $15^{\text {th }}$ Int. Symposium on District Heating and Cooling, Seoul, South Korea.

Robineau, J.-L., Page, J., Maréchal, F., 2016, A method for taking into account seasonal storage in a district energy system optimisation problem. $15^{\text {th }}$ Int. Symposium on District Heating and Cooling.

Sancho-Tomás, A., Sumner, M., Robinson, D., 2017, A generalised model of electrical energy demand from small household appliances. Energy Build., vol. 135, pp. 350-366.

Skarbal, B., Peters-Anders, J, Malik Faizan, A., Agugiaro, G., 2017, How to pinpoint energy-inefficient buildings? An approach based on the 3D city model of Vienna. ISPRS Ann. Photogramm. Remote Sens. Spatial Inf. Sci. $2^{\text {nd }}$ Int. Conf on Smart Data and Smart Cities 2017, Puebla, Mexico, 4-6 October 2017.

Tardioli, G., Kerrigan, R., Oates, M., O‘Donnell, J., Finn, D., 2015 , Data Driven Approaches for Prediction of Building Energy Consumption at Urban Level. Energy Procedia, vol. 78, pp. 33783383.

United Nations, 2014, World Urbanization Prospects: The 2014 Revision, Highlights. United Nations, Department of Economic and Social Affairs, Population Division, ST/ESA/SER.A/352.

Urbact, 2013, Cities of Tomorrow - Action Today. URBACT II Capitalisation. Building energy efficiency in European cities.

Wang, K., Robinson, D., 2016, Towards the generalized co-simulation of urban energy systems. Urban Transitions Global Summit 2016, Shanghai, China (in press)

Wate, P., Coors, V., 2015, 3D Data Models for Urban Energy Simulation. Energy Procedia, vol. 78, pp. 3372-3377.

Wate, P., Rodrigues, P., Duminil, E., Coors, V., 2016, Urban energy simulation based on 3D city models: a service-oriented approach. ISPRS Ann. Photogramm. Remote Sens. Spatial Inf. Sci., IV-4-W1, pp. $75-80$.

\section{REFERENCES FROM WEBSITES (15 MAY 2017)}

CESIUM, https://cesiumjs.org/

CINERGY, http://www.ci-nergy.eu

ENE-ADE, http://en.wiki.energy.sig3d.org

INSP-BDG, http://inspire.ec.europa.eu/Themes/126/2892

INTEGRCITY, http://integrcity.epfl.ch

OCEN, http://ge.ch/energie

OGD-W, https://open.wien.gv.at

SC-W,

https://smartcity.wien.gv.at/site/files/2014/09/SmartCityWien_Framew orkStrategy_english_doublepage.pdf

SEP-W, https://www.wien.gv.at/stadtentwicklung/energie/pdf/sependbericht.pdf

SITG, http://ge.ch/sitg

UTN-ADE, http://en.wiki.utilitynetworks.sig3d.org 